\title{
Life Support Systems for Lunar Landers
}

\author{
Molly Anderson
}

NASA

Copyright (C 2008 SAE International

\begin{abstract}
Engineers designing life support systems for NASA's next Lunar Landers face unique challenges. As with any vehicle that enables human spaceflight, the needs of the crew drive most of the lander requirements. The lander is also a key element of the architecture NASA will implement in the Constellation program. Many requirements, constraints, or optimization goals will be driven by interfaces with other projects, like the Crew Exploration Vehicle, the Lunar Surface Systems, and the Extravehicular Activity project. Other challenges in the life support system will be driven by the unique location of the vehicle in the environments encountered throughout the mission.
\end{abstract}

This paper examines several topics that may be major design drivers for the lunar lander life support system. There are several functional requirements for the lander that may be different from previous vehicles or programs and recent experience. Some of the requirements or design drivers will change depending on the overall Lander configuration. While the configuration for a lander design is not fixed, designers can examine how these issues would impact their design and be prepared for the quick design iterations required to optimize a spacecraft.

\section{INTRODUCTION}

The lunar lander will be important part of NASA's execution of the Vision for Space Exploration. In order to explore the lunar surface, landing successfully and returning safely are clearly critical steps. The capabilities of the lander will determine when locations on the lunar surface can be explored. The lunar lander is also a forerunner of vehicles that will eventually bring explorers to the Martian surface.

For the lunar campaign, three types of missions are expected for the lunar lander. The first mission is a lunar Sortie where the lander operates independently of other resources on the lunar surface. The lunar Sortie mission will be an important part of lunar science and exploration work. This mission could deliver crewmembers to many different locations on the lunar surface for the maximum range of exploration activities and support intravehicular activity (IVA) and extravehicular activity (EVA) for the crew. The second mission is a delivery of crew to the lunar Outpost, sometimes referred to as a "Down and Out" mission for the lander. The lander again delivers the crew to the lunar surface, but in this case they almost immediately transfer to the lunar Outpost or some other transport vehicle. For current design work, this mission is assumed to go to a polar location. The lander is left unmanned in a dormant state until it is time for the crew to return home, which could be up to 210 days. The third mission is cargo delivery, which has minimal relevance to the design of life support systems. NASA's goal is to have a single coe lander design to accomplish all three missions.

Preliminary design work is in progress for the lunar lander, including the life support systems, as part of NASA's Altair Project. Experience with the Orion Project Crew Exploration Vehicle (CEV), however, has clearly demonstrated that at this stage of development many concept changes are likely still ahead and decisions are far from final. This paper focuses on some of the key requirements and issues that have been identified to date from that work that will be important to any lunar lander design.

\section{MAJOR DESIGN DRIVERS}

In many ways designers of a lander life support system will have to consider the same issues that designers of any other manned vehicles have dealt with. In many cases, the most obvious parallels are drawn with the CEV since the vehicles have many of the same specifications for human related requirements and many of the same technological options for implementation since their development cycles overlap. However, there are some key design drivers that depend on the mission the crewmembers must perform on the lunar surface and on the way the lander fits into an entire Exploration architecture. The life support system may be 
constrained or asked to design certain things to help the performance of the overall lander even if they do not seem optimize the life support system.

THE BASICS - The basic needs of human beings do not vary much depending on their location. As a result, the key drivers for the life support system for this vehicle are the crew size and mission duration. The lander is designed to support up to 4 crewmembers (CARD 2007). Unlike the CEV, there are no 6 crewmember missions planned.

There are several different durations of mission segments that impact the design. Unlike in Apollo, the lander is launched from Earth separately from the crew, and some loiter time in Earth orbit is required. Unless the life support system has its own cryogenic fluid storage resources, this is not expected to be a major driver for lander life support system design. After the CEV and Lander dock and leave Earth orbit together, the transit from the Earth to the moon is several days long, but the CEV is the primary vehicle for crew support.

The most important phase of the lander's mission begins after it performs the propulsive maneuver for lunar orbit insertion. The lander and CEV undock and the lander descends to the lunar surface entirely on its own resources. The durations on the surface are the biggest drivers for the life support system, but the issues are very different depending on whether it is a Sortie mission for a mission to the Outpost.

A Sortie mission has a seven day stay on the surface, and the lander provides life support and EVA resources for the crew members during this time. One requirement on a Sortie mission is the ability to perform "split operations". This allows part of the crew to be in the lander's habitable volume in a shirtsleeve environment, while the rest of the crew uses an airlock to perform EVAs.

In a mission to the Outpost, the crew exits the vehicle within a few hours of landing, and the lander stays dormant and unmanned for up to 210 days, with minimal power provided by the Lunar Surface Systems. There are no split operations required for this vehicle, but it also may not necessarily have an airlock as part of the vehicle.

After the surface operations phase of a Sortie mission is complete, or after the crew returns to the vehicle after a mission to the Outpost, the ascent stage will return the crew to the CEV, which is waiting in orbit. Once the lander docks with the CEV and hatches between the two are opened, the CEV again becomes the primary vehicle for crew support. After a short time, the vehicles undock, and the abandoned lander ascent stage will by disposed of by crashing onto the lunar surface.

NEW FUNCTIONS - Missions to the lunar surface will require functionality not required for NASA's most recent vehicles designed for missions in microgravity and Low Earth Orbit (LEO). Many of these requirements are due to EVA activities.

Lunar Dust - As crewmembers exit and return to the lander, they will inevitably bring some lunar regolith or lunar dust into the vehicle. The medical community is concerned about the negative health effects that this dust could have on the crewmembers if inhaled (HSIR 2007). It can be generally irritating to the crew's eyes, noses, and skin. And it may damage equipment through abrasion, fouling surfaces, or other methods.

Before the dust enters the vehicle, the life support system will need to work in concert with structural systems like airlocks and the EVA systems to prevent the dust from entering the vehicle. Good design of the EVA suits will be important, but good design of the airlock systems may be more important. Airlock repressurization and ventilation systems should be designed to be as helpful as possible. Using repressurization gas to blow dust off of surfaces into capture areas, and ensuring that ventilation systems draw dirty air through filters and return clean air to the airlock are good examples of helpful ways of performing functions that must be provided anyway.

The life support system will have a primary role in removing any of the dust that enters the vehicle. Control of airborne particulates is not a new requirement for spacecraft Ifie support systems. However, the particle size, allowable concentration in the vehicle air, load of particles brought into the vehicle, and the properties of those particles may all be significantly different than the usual dust and lint previous systems were designed to control.

Atmosphere - The vehicles included in the Constellation Program have many conflicting goals when trying to determine the best atmospheric makeup for the vehicle (Campbell 2006). The lander is driven by the need to dock with the CEV, and the frequent EVAs planned as part of lunar surface exploration. The CEV and lander are expected to have an atmosphere of $70.3 \mathrm{kPa}(10.2$ psia) when docked, without exceeding 30\% $\mathrm{O}_{2}$. Because the first flight of the lander is later than that of the CEV, more time is expected for materials certification. The lander also does not have to carry crew or cargo to the International Space Station. As a result, the lander is allowed have oxygen concentrations of up to $34 \%$ when not sharing atmosphere with the $\mathrm{CEV}$. The total pressure of the vehicle can be reduced to near $55 \mathrm{kPa}$ (8 psia) to reduce the partial pressure of nitrogen in the atmosphere and reduce the risk of decompression sickness when crewmembers perform EVAs.

These choices create two requirements for pressure control systems in the lunar lander. First, obviously, multiple set points must available for control. Second, the systems must be highly accurate to operate at the low pressure high oxygen concentration atmosphere 
setpoint in order to manage flammability risk and not allow the partial pressure of $\mathrm{O}_{2}$ to drop too low. As an additional issue, there is very little margin in the partial pressure of oxygen and the vehicle volume will be small. This means that in the event of leaks or other unexpected depressurizations, there is not much margin before the oxygen in the vehicle is insufficient. The pressure at which emergency systems actuate will be very close to the nominal vehicle pressure, which has the potential to be problematic.

Supporting EVA - Nearly every NASA vehicle since Gemini has had to support EVAs of some kind, so it is not truly a new function. But two new configurations of spacesuits will be developed within the Constellation Program. The interfaces and requirements of these suits will be different from recent experience with Shuttle and International Space Station era experience with Extravehicular Mobility Unit (EMU) or Advanced Crew Escape Suits (ACES).

The EVA system puts some basic requirements on the vehicle life support system even before crewmembers exit the vehicle. The crewmembers are assumed to wear suits without Portable Life Support Systems (PLSSs) during descent, ascent, and any docking or undocking maneuvers to protect them from accidental depressurization. Much like the CEV, the lander must provide life support functions including air revitalization (AR) and liquid cooling to the suits via umbilical during these periods.

The requirement for split operations leaving some crewmembers in the cabin while others go out for an EVA drives the life support system architecture. The crewmembers inside the cabin do not need to utilize liquid cooling, so the same system used during ascent and descent may be available for use. When crewmembers are preparing to go out for an EVA, they must eventually purge their spacesuits to $100 \% \mathrm{O}_{2}$. The crewmembers inside the cabin volume, however, remain in a mixed $\mathrm{O}_{2}$ and $\mathrm{N}_{2}$ atmosphere. The same life support system cannot process air for both at the same time. And unlike the EMU, the next generation space suit will require vacuum for some functions to operate. The designers of the lander have the option of either providing additional hardware to provide air revitalization for crewmembers still in a pressurized airlock, or providing vacuum access as a resource to the suits.

The lander life support system is also the source or conduit for resources needed to resupply the EVA PLSSs so that the crew can conduct multiple EVAs. Water and oxygen are the consumables required from the life support system. Several concerns will be important when providing these resources.

One important issue is the condition of the consumables. For water, the current interface requires that the same feed of water provide liquid cooling recirculation and refill PLSS water tanks. This may require larger sizes of heat exchangers since a high flowrate of water is required to refill the PLSS tanks, but it must all be cooled. For oxygen, efficient storage in the PLSS requires either high pressures or cryogenic fluid. High pressure gas I the currently assumed baseline. In order to quickly delivery high pressure gas, it must begin at an even higher pressure. The lander must therefore either have significant quantities of very high pressure gas at > $21000 \mathrm{kPa}$ (3000 psia), or a pressurization system.

The developers of the next generation EVA system also seek to achieve certain targets for a Work Efficiency Index (WEI). Essentially, the goal of using the WEI is to reduce the time to prepare for an EVA compared to the time doing useful exploration. This is most likely to impact the delivery rate. If the resources cannot be safely or reasonably provided quickly to a crewmember preparing for EVA, then the PLSSs will have to be charged overnight.

Interfaces to Other Vehicles - Another difference between the new lander and previous vehicles is that it will be interfacing with new vehicle or habitat systems.

The relationship between the lander and the CEV is unique because they have development cycles that overlap, yet they are launched on separate launch vehicles and do not have a shared launch mass problem. Determining which vehicle provides which type of resource or environmental control while they are docked is a complicated optimization problem. Handling lunar dust is also an issue that concerns both vehicles, because any dust the lander brings to orbit could enter the CEV atmosphere. Providing clean, cool, dry air to the lander requires a lower flow rate from the CEV to maintain habitable conditions than drawing lander air into the CEV ARS and returning CEV cabin air to the lander. However, providing clean air to the lander means that lander atmosphere returns to the CEV unfiltered through the hatchway.

The other key relationship the lander has is with the Lunar Surface Systems. Most concepts do not have the lander docking directly with a habitat on the lunar surface. But any resources left behind by the lander will be considered available for scavenging for later use. In the lander, hydrogen and oxygen from descent stage engines can be used to generate critical stores of water. Depending on how long this water must be stored, freezing or microbial growth may be concerns, and the water may have to be processed by recycling hardware anyway to achieve potability. If water recycling is part of the life support systems on the lunar surface as expected, wastewater, or even wet trash and other solid wastes may be useful resources. Finally, spare parts are another way that pieces of the lander may be used in other areas of the Lunar Surface Systems. The availability of spare life support system parts is highly dependent on the configuration of the lunar lander. Items left behind on the surface with the descent stage will likely be close to the Outpost location and accessible. Items disposed of when the ascent stage is 
disposed of will be farther away and may not be in any condition suitable for reuse.

LANDER CONFIGURATION - The mass of the life support system is relatively small compared to systems like propulsion and structure, and the rest of the lunar lander as a whole. Major configuration decisions may be made to benefit the entire design that make things more complicated for the life support system. Examples of this are the configuration and connection of habitable pressure vessels, and airlock design.

Pressure Vessels - To accomplish the reference missions set out, three categories of pressurized volumes are required. An ascent vehicle must return the crew from the surface to the CEV, a habitable volume must be provided with food, hygiene, and sleeping accommodations for the 7-day Sortie mission, and an airlock has been listed as a requirement for split operations and dust mitigation. However these do not necessarily have to be three separate volumes.

Any mass that ascends from the lunar surface will be the most expensive of any in the lunar campaign due to all the propulsion required to deliver and return it. As a result, if the ascent vehicle and the habitable volume are not the same, the ascent vehicle can be optimized for minimum mass for the short duration. The habitat also does not have to provide life support via umbilical to suited crewmembers. The habitable volume would not be required at all for missions to the Outpost. If they are the same volume, then an important mass vs. life cycle cost trade needs to be made. The vehicle could have the same systems installed regardless of mission for simplicity of construction. Certain systems or components could be removed if they were not required for the mission to the Outpost. Or the installed systems could each be optimized for mass based on their duration requirements and be significantly different, and essentially create two separate designs to be developed and certified.

Airlocks - The Apollo Lunar Module did not have an airlock, but with four crewmembers and Apollo experience with lunar dust, the new lander will be required to have an airlock at least for Sortie missions. Airlocks also reduce the loss of consumables. There are several options for airlocks as well that each provide unique options.

Simply including an airlock volume means that when part of the crew goes on an EVA, the gas inside the primary habitable volume is not lost. But airlocks also provide an opportunity to reclaim the gas inside the airlock by compressing it back into the main volume or a storage tank. For the short lunar lander missions, and the lower pressure atmospheres to be used, the mass and power of the compression system may not be worth the mass of gas saved. However, if a common airlock design, or even reuse of the same vessel, is desired between the lander and the Outpost, those interfaces may have to be designed anyway.
The airlock concept is also very important to the life support system. The two most likely options are a conventional airlock, separate with a hatch, or a suitlock, which combines the suitport hatch for easy rear-entry suit donning and dust barriers with an exterior hatch to leave the suits in a pressurized environment when not in use. In a conventional airlock, more dust may be able to enter the habitable volume when the hatch is opened. Procedures for suit or airlock cleaning to prevent this transfer may need to be more elaborate. However, ventilation can be performed with a drag-through hose that does not require hard fluid connections between the two vessels if they ever have to separate. A suitlock using the suitport type interfaces will likely provide a more effective dust barrier, especially since the amount of maintenance should be low on a short Sortie mission.

Propellants and Other Fluid Resources - The mass of the propulsion system and propellant is always one of the largest and often the largest portion of spacecraft mass. Optimization of that system is clearly more important to integrated performance than the life support system! However, if the vehicle has made choices in the propulsion system to use oxygen based systems, such as the liquid oxygen (LOx) - hydrogen system required for the Altair lander, that resource could be shared with life support. This adds new requirements to the vehicle, however, as the gas quality requirements for the propulsion system and the life support system will need to be combined to create a new quality standard. Delivery pressures are another area that are important. Higher pressure feeds of boiloff from cryogenic fluid boiloff would be useful to life support to have smaller lines and ease of delivery, but the structural requirements on the tanks have a huge impact since the propulsion tanks are very large.

The power system is another area that can benefit from fluids originally provided by propulsion. In an oxygenhydrogen system, the same reactants can be used for a fuel cell based power system as well as propulsion. Fuel cell systems create water which is one of the largest consumable masses required by the life support system. Having to manage constant production and intermittent use of water and maintain potability in the system will be very different for the lander than for the CEV, where stored water is the only source available.

\section{CONCLUSION}

Many of the requirements, interfaces, or issues that will drive the design of the lunar lander are similar to issues seen in previous vehicles. But the combination of all of them mean that NASA's new lunar lander will be different in important ways from previous vehicles. Interfaces and requirements from the EVA Project are an important source of these requirements. Because the new suits are still under development, developers of lander life support systems need to be flexible and prepared for possible changes. Also, because the lander fits into the middle of NASA's Exploration 
architecture, optimization at a system level may also result in changes in requirements. Developers who understand how outside drivers impact their design and are prepared with alternatives will be most successful in the design of the new lunar lander.

\section{REFERENCES}

1. Campbell, Paul D. "Recommendations for Exploration Spacecraft Internal Atmospheres: The Final Report of the NASA Exploration Atmospheres Working Group," NASA JSC-63309, Houston, January 2006.

2. "Constellation Architecture Requirements Document (CARD), Revision A, Change 001," CXP 70000, NASA, November 2, 2007

3. "Human-System Integration Requirements (HSIR), Revision A, Change 001," CXP 70024, NASA, October 30, 2007.

\section{CONTACT}

The author can be contacted via email at molly.s.anderson@nasa.gov. 